\title{
BMJ Open Simvastatin add-on to escitalopram in patients with comorbid obesity and major depression (SIMCODE): study protocol of a multicentre, randomised, double-blind, placebo-controlled trial
}

Christian Otte (D) , ${ }^{1}$ Woo Ri Chae (D) , ${ }^{1}$ Jan Nowacki, ${ }^{1}$ Michael Kaczmarczyk, ${ }^{1}$ Dominique Piber, ${ }^{1}$ Stefan Roepke, ${ }^{1}$ Stefanie Märschenz (1) , ${ }^{2}$ Sandra Lischewski, ${ }^{2}$ Sein Schmidt, ${ }^{3}$ Barbara Ettrich, ${ }^{4}$ Hans Joergen Grabe, ${ }^{5}$ Ulrich Hegerl, ${ }^{6}$ Kim Hinkelmann, ${ }^{7}$ Tobias Hofmann, ${ }^{7}$ Deborah Janowitz, ${ }^{5}$ Klaus Junghanns, ${ }^{8}$ Kai G. Kahl, ${ }^{9}$ Jan Philipp Klein (1) , ${ }^{8}$ Tillmann H. C. Krueger, ${ }^{9}$ Gregor Leicht, ${ }^{10}$ David Prvulovic, ${ }^{11}$ Andreas Reif, ${ }^{11}$ Daniel Schoettle, ${ }^{10}$ Maria Strauss, ${ }^{4}$ Anna Westermair, ${ }^{8}$ Tim Friede (D) , ${ }^{12}$ Stefan M Gold (D) ${ }^{1,7,13}$

To cite: Otte C, Chae WR, Nowacki J, et al. Simvastatin add-on to escitalopram in patients with comorbid obesity and major depression (SIMCODE): study protocol of a multicentre, randomised, double-blind, placebocontrolled trial. BMJ Open 2020;10:e040119. doi:10.1136/ bmjopen-2020-040119

\section{- Prepublication history and} additional material for this paper is available online. To view these files, please visit the journal online (http://dx.doi.org/10. 1136/bmjopen-2020-040119).

Received 05 May 2020 Revised 28 0ctober 2020 Accepted 08 November 2020

Check for updates

(C) Author(s) (or their employer(s)) 2020. Re-use permitted under CC BY-NC. No commercial re-use. See rights and permissions. Published by BMJ.

For numbered affiliations see end of article.

Correspondence to

Christian 0tte;

christian.otte@charite.de

\section{ABSTRACT}

Introduction Major depressive disorder (MDD) and obesity are both common disorders associated with significant burden of disease worldwide. Importantly, MDD and obesity often co-occur, with each disorder increasing the risk for developing the other by about $50 \%-60 \%$. Statins are among the most prescribed medications with well-established safety and efficacy. Statins are recommended in primary prevention of cardiovascular disease, which has been linked to both MDD and obesity. Moreover, statins are promising candidates to treat MDD because a meta-analysis of pilot randomised controlled trials has found antidepressive effects of statins as adjunct therapy to antidepressants. However, no study so far has tested the antidepressive potential of statins in patients with MDD and comorbid obesity. Importantly, this is a difficult-to-treat population that often exhibits a chronic course of MDD and is more likely to be treatment resistant. Thus, in this confirmatory randomised controlled trial, we will determine whether add-on simvastatin to standard antidepressant medication with escitalopram is more efficacious than add-on placebo over 12 weeks in 160 patients with MDD and comorbid obesity.

Methods and analysis This is a protocol for a randomised, placebo-controlled, double-blind multicentre trial with parallel-group design (phase II). One hundred and sixty patients with MDD and comorbid obesity will be randomised 1:1 to simvastatin or placebo as add-on to standard antidepressant medication with escitalopram. The primary outcome is change in the Montgomery-Åsberg Depression Rating Scale (MADRS) score from baseline to week 12 . Secondary outcomes include MADRS response (defined as $50 \%$ MADRS score reduction from baseline), MADRS remission (defined as MADRS score $<10$ ), mean change in patients' self-reported Beck Depression Inventory (BDI-II) and mean change in high-density lipoprotein, low-density lipoprotein and total cholesterol from baseline to week 12 .

\section{Strengths and limitations of this study}

- This is a confirmative randomised controlled trial to examine the antidepressive potential of simvastatin as add-on therapy in patients with major depressive disorder (MDD) and comorbid obesity.

- Importantly, depressed patients with obesity are a difficult-to-treat population that often exhibits a chronic course of MDD and treatment resistance.

- This trial is not designed to examine long-term effects of add-on statin therapy (beyond 12 weeks of follow-up).

- This trial is not powered to explore effects in subgroups (eg, patients with type 2 diabetes mellitus).

Ethics and dissemination This protocol has been approved by the ethics committee of the federal state of Berlin (Ethik-Kommission des Landes Berlin, reference: 19/0226-EK 11) and by the relevant federal authority (Bundesinstitut für Arzneimittel und Medizinprodukte (BfArM), reference: 4043387). Study findings will be published in peer-reviewed journals and will be presented at (inter)national conferences.

Trial registration numbers NCT04301271, DRKS00021119, EudraCT 2018-002947-27.

\section{INTRODUCTION}

Scientific background and study rationale The WHO's global burden of disease (GBD) study demonstrated that of all diseases worldwide, major depressive disorder (MDD) is among the top five with regard to years lived with disability ${ }^{1}$ and by far the leading cause of disability resulting from all psychiatric disorders. ${ }^{2}$ The 12 months 
prevalence of MDD in adults according to the World Mental Health Survey is approximately 6\%, which is comparable to estimates from other (inter-)national surveys. ${ }^{3}$ Mortality rates in MDD are twice as high as in the general population and translate to a reduced life expectancy of approximately 14.0 years in men and 10.1 years in women based on a cohort study of all Danish residents aged 15 or older. ${ }^{4}$

According to the GBD study, the overall prevalence of obesity in adults was $12.0 \%$ in 2015 and ranged from $1.6 \%$ in Vietnam to $34.9 \%$ in Egypt. ${ }^{5}$ According to the latest global estimates of the WHO, $11 \%$ of adult men and $15 \%$ of adult women were obese in 2016 and the prevalence ranged from 29\% among the Region of the Americas to $2 \%$ in some of the West African countries. ${ }^{6}$ In the German adult population (age range: $18-79$ years), the prevalence of obesity is $23.3 \%$ in men and $23.9 \%$ in women, respectively. ${ }^{7}$ In adult patients with obesity, mortality rates are elevated (HR 1.64, CI 1.61 to 1.67) and they increase in relation to body mass index (BMI) as recently confirmed in a meta-analysis of 239 studies from four continents. ${ }^{8}$ In the GBD study, obesity was estimated to cause 3.4 million deaths in the general population, and accounted for $3.9 \%$ of years of life lost, and $3.8 \%$ of disability-adjusted life years worldwide. ${ }^{9}$

MDD and obesity are both linked to a higher risk of cardiovascular disease and stroke, further increasing their public health and economic impact. Importantly, MDD and obesity frequently co-occur and the presence of one condition increases the risk for developing the other by approx. $50 \%-60 \% .{ }^{10}$

Statins (3-hydroxy-3-methylglutaryl coenzyme A reductase inhibitors) are among the most prescribed medications worldwide with well-established safety and efficacy. Recent guidelines recommend the use of statins in primary prevention of cardiovascular disease, ${ }^{11}$ which has been linked to both MDD and obesity. Moreover, statins are promising candidates to treat MDD. Evidence for antidepressive effects of statins has accumulated from four independent lines of research: animal studies ${ }^{12}$ meta-analysis of prospective cohort studies, ${ }^{13}$ meta-analysis of pilot controlled trials ${ }^{14}$ and a nationwide cohort study. ${ }^{15}$ However, no randomised controlled study so far has tested the antidepressive potential of statins in patients with MDD and comorbid obesity. Importantly, this is a difficult-to-treat population that often exhibits a chronic course of MDD and is more likely to show treatment resistance to standard pharmacological therapy. ${ }^{16}{ }^{17}$ Depressed patients who are overweight or obese responded significantly slower to antidepressant treatment compared with normal-weight patients. ${ }^{18}$ In a recent scoping review, excess body weight predicted nonresponse to treatment with antidepressants. ${ }^{19}$ Given the enormous public health impact of MDD and obesity and given the evidence of antidepressive effects of statins, a controlled trial of adjunct statin treatment in comorbid patients appears warranted.

\section{STUDY AIMS AND HYPOTHESIS}

\section{Primary}

To examine whether add-on $40 \mathrm{mg} / \mathrm{d}$ simvastatin to standard antidepressant medication (escitalopram $20 \mathrm{mg} / \mathrm{d}$ ) (SIM+ES) improves depression to a greater extent than adjunct placebo (PL+ES) in patients with MDD and comorbid obesity over a period of 12 weeks. We hypothesise that SIM+ES will improve depression to a greater extent than PL+ES in patients with comorbid obesity and major depression.

\section{Secondary}

To examine whether SIM+ES improves response rates, remission rates, self-reported depression, patients' impression of change, clinicians' impression of severity and change, quality of life, social functioning, lipid values and immune function and cellular metabolism to a greater extent than PL+ES in patients with major depression and comorbid obesity. We hypothesise that SIM+ES will improve (a) response rates and remission rates, (b) patients' impression of change, clinicians' impression of severity and improvement, quality of life, social functioning, self-report depression, (c) lipid values and (d) immune function and cellular metabolism to a greater extent over 12 weeks than PL+ES in patients with comorbid obesity and major depression.

\section{METHODS}

This protocol has been prepared in accordance with the Standard Protocol Items: Recommendations for Interventional Trials (SPIRIT) 2013 statement. ${ }^{20}$ Please see online supplemental table 1 for the SPIRIT checklist.

\section{Study design and sample}

The study will include 160 patients divided into two treatment groups and recruited from several centres (public academic hospitals/departments) located in Germany. Group 1 will receive placebo add-on to escitalopram $(\mathrm{PL}+\mathrm{ES})$ and group 2 will receive simvastatin add-on to escitalopram (SIM+ES). For an overview of the study design, please see figure 1 .

Patients will be recruited from depression and obesity outpatient clinics and inpatient wards from each study site. In addition, we will use existing and established networks of all sites within the psychiatric community to include depressed out-patients from general practitioners and psychiatrists. Thus, we will ensure high generalisability of our study population. There will be no specific sex distribution as no sex specific differences concerning efficacy and safety of simvastatin are expected. The first study sites have been initiated in March 2020 and the study is expected to end in January 2023.

\section{Eligibility criteria and assessment}

The main inclusion criteria for the SIMCODE trial are as follows: (i) written informed consent; (ii) major depressive episode according to DSM 5 (Diagnostic and Statistical Manual of Mental Disorders fifth Edition); (iii) 


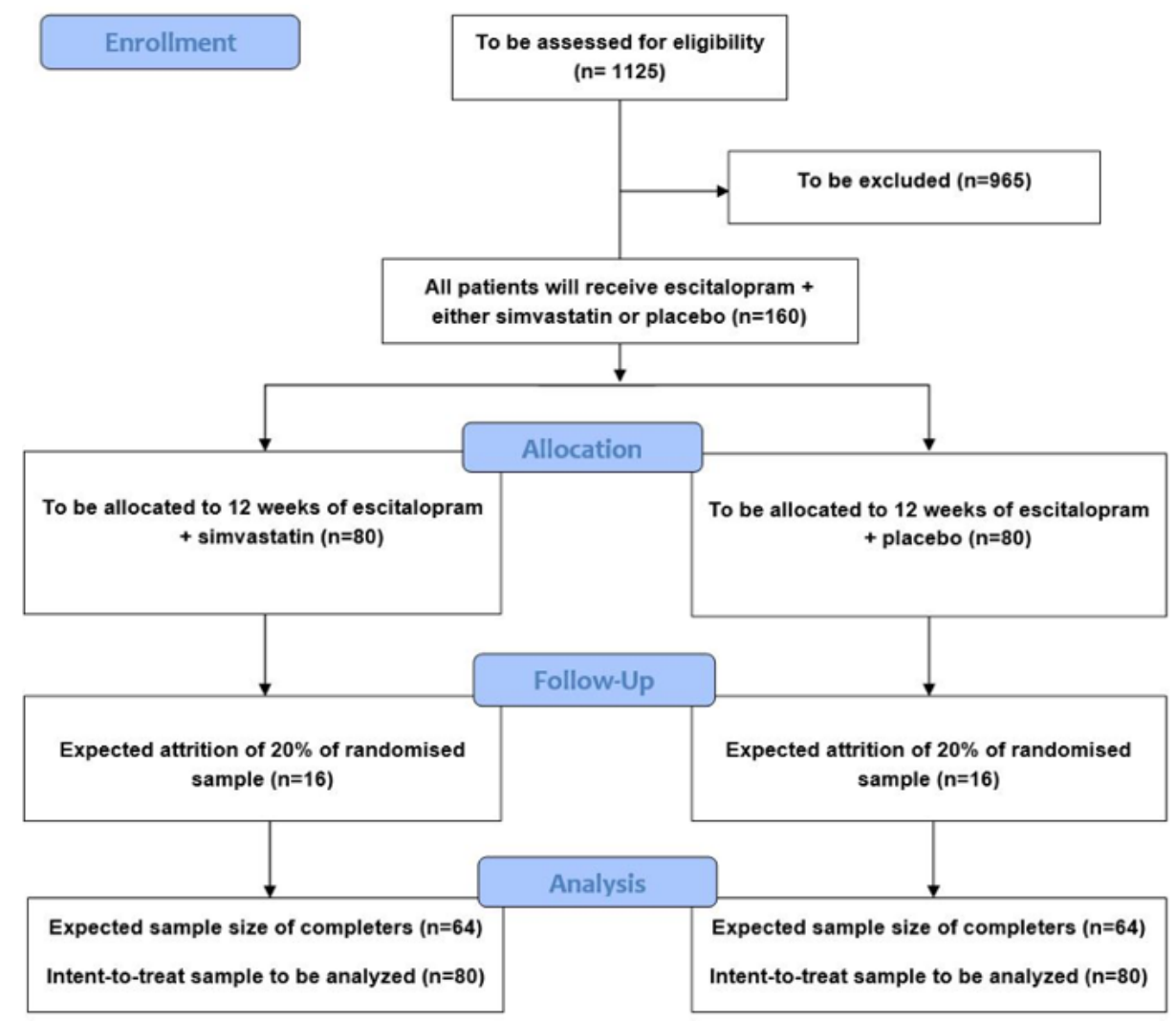

Figure 1 Study flow chart according to CONSORT. We assume that we need to screen $n=1125$ patients aged 18-65 with major depression according to Diagnostic and Statistical Manual of Mental Disorders five and obesity (body mass index $\geq 30$ ) for eligibility at eight recruiting centres to randomise $n=160$ participants. The sample size and assumed dropout rate (20\%) is conservatively based on three earlier pilot RCTs of add-on statin to SSRI treatment in depressed patients. CONSORT, Consolidated Standards of Reporting Trials; SSRI, selective serotonin reuptake inhibitors; RCT, randomised controlled trials. ${ }^{23} 3132$

Montgomery-Åsberg Depression Rating Scale (MADRS) score $\geq 18$; (iv) BMI $\geq 30$; (v) age between 18 and 65 years ( $\geq 18$ und $\leq 65)$; (vi) in case of non-psychotropic medication: stable pharmacological medication for at least 14 days prior to study entry; (vii) no antidepressant intake during the last 7 days prior to study entry (discontinuation of effective medication to enable study participation is prohibited); (viii) no prior treatment with escitalopram in index episode; (ix) less than three trials with antidepressants in index episode; (x) no treatment with ketamine, electroconvulsive therapy (ECT) or other stimulatory treatments in index episode; (xi) none of the following disorders: schizophrenia, schizoaffective disorder and bipolar disorder. In addition, patients with any severe, unstable general medical condition or contraindications for simvastatin or escitalopram will not be included in the study.

Main exclusion criteria are as follows: (i) current use of statins; (ii) current use of antidepressants; (iii) acute suicidal tendencies (MADRS Item 10>4); (iv) pregnancy, breastfeeding or women with childbearing potential without acceptable form of contraception (defined as Pearl index $<1$ ); (v) current use of psychotropic medication (eg, antipsychotics, anticonvulsants, lithium or St. John's Wort) except for benzodiazepines, non-benzodiazepines and opiates (vi) clinically significant abnormalities in 12-lead ECG (eg, corrected Q-T interval prolongation $\geq 500 \mathrm{~ms}$ or increase $\geq 60 \mathrm{~ms}$ from baseline visit). In addition, there are several more exclusion criteria including medication that is contraindicated with simvastatin or escitalopram. Please see online supplemental table 2 for complete inclusion and exclusion criteria.

A screening visit will be carried out to establish eligibility and to obtain informed consent. After informed consent, we will assess the severity of depressive episode using MADRS, duration of the index episode, number of episodes, previous treatment as requested by the guideline of the European Medicines Agency on clinical trials in depression. ${ }^{21}$ During the screening visit, several other procedures will be performed including a physical examination, Mini International Neuropsychiatric Interview (MINI; German Translation V.7.0.2), ${ }^{22}$ measurement of body weight and height for determination of BMI, ECG and safety laboratory including pregnancy test for women.

To ensure that the study is conducted according to the study protocol, to current laws and to general guidelines, a training of all investigators/subinvestigators have been conducted prior to initiation. The trial will be initiated by the principal investigator $(\mathrm{PI})$ team $(\mathrm{CO}, \mathrm{WRC})$ and a trial monitor at each site before patient enrolment at that site begins. 

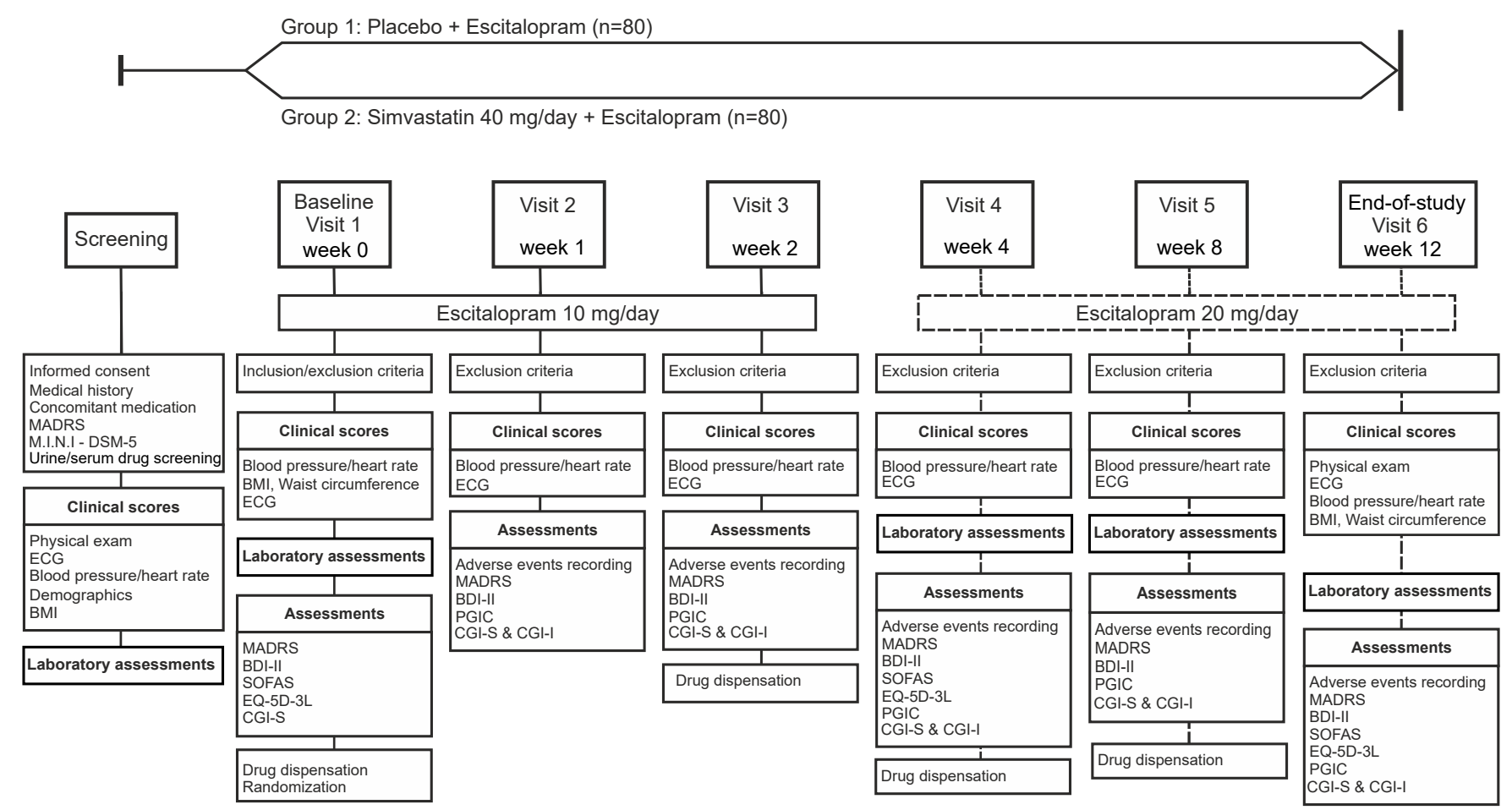

Figure 2 Scheme of intervention in SIMCODE study. Overview of design and procedures of the SIMCODE study. BDI-II, BeckDepressions-Inventar II; BMI, body mass index; CGI-I, Clinicians' Global Impression of Improvement; CGI-S, Clinicians' Global Impression of Severity of illness; EQ-5D-3L, Generic Quality of Life Questionnaire; MADRS, Montgomery-Åsberg Depression Scale; MINI, Mini International Neuropsychiatric Interview; PGIC, Patient Global Impression of Change; SOFAS, Social and Occupational Functioning Assessment Scale.

\section{Intervention}

Each patient in this study will be treated with escitalopram, a well-established standard-antidepressant, which is readily available because it is commonly used as generic drug in Germany. Add-on placebo serves as the control condition for adjunct simvastatin $(40 \mathrm{mg} / \mathrm{d})$. Simvastatin and placebo will be provided by the pharmacy of the Charité-Universitätsmedizin Berlin and will be administered in a double-blind fashion. Escitalopram will be provided by the pharmacy of the Charité, stored and dispensed at each study site in a non-blinded fashion. Patients will receive simvastatin or placebo orally in a fixed dosage of $40 \mathrm{mg}$ once a day at bedtime. Patients will receive $10 \mathrm{mg}$ escitalopram orally in a fixed dosage of 10 mg once a day in the morning for the first 2 weeks, then increased to $20 \mathrm{mg}$ once a day in the morning until the end of study. Drug dispensation will take place at baseline (visit 1), at visit 3 (only escitalopram), visit 4 and visit 5 . See figure 2 for scheme of intervention.

\section{Rationale for statin choice and dosage}

Among the available stains, we decided to use simvastatin (SimvaHEXAL) in a dosage of $40 \mathrm{mg} / \mathrm{d}$ based on the following considerations: simvastatin was used in this dose in one of three randomised controlled trials (RCTs) showing evidence versus placebo as adjunct treatment to selective serotonin reuptake inhibitors (SSRIs) ${ }^{23}$ it was superior to atorvastatin in depressed patients with heart disease $;{ }^{24}$ it passes the blood-brain barrier ${ }^{25}$ and it is available as generic drug (costs for $40 \mathrm{mg}$ /d simvastatin: around 100 EUR per year). We decided against using a dose of $80 \mathrm{mg} / \mathrm{d}$ simvastatin due to the increased risk of muscle injury and the corresponding warning from the US Food and Drug Administration ${ }^{26}$ (see 3.8 Safety).

\section{Rationale for antidepressant choice and dosage}

All depressed patients enrolled in the trial will be continuously treated with a gold standard antidepressant (escitalopram) as described by the German National Disease Management Guideline ('Nationale Versorgungsleitlinie') for the treatment of MDD. For escitalopram, a dose-response relationship has been suggested. Especially for patients with severe depression, the high standard dose of $20 \mathrm{mg} / \mathrm{d}$ was shown to be superior compared with $10 \mathrm{mg} / \mathrm{d} .{ }^{27}$ A fixed-dose of $10 \mathrm{mg} / \mathrm{d}$ escitalopram for the entire study duration of 12 weeks would therefore not allow the optimal treatment for some patients. Thus, we consider this option ethically not justifiable. A dose increase from 10 to $20 \mathrm{mg} / \mathrm{d}$ based on individual patient response would however interfere with comparability of the SIM+ES group and PL+ES group, because a strong decrease in depressive symptoms in the SIM+ES group compared with PL+ES group could potentially be masked by a higher percentage of escitalopram dose escalation in the PL+ES group. Therefore, we will increase escitalopram to $20 \mathrm{mg} / \mathrm{d}$ after giving a low standard dose of 10 $\mathrm{mg} / \mathrm{d}$ in the first 2 weeks. In case of adverse reactions 
related to escitalopram, we will follow the instructions for dose adjustment described in 3.8 Safety.

Patients are instructed to return unused medication and compliance will be determined by count of the remaining pills and patients will be considered compliant if they report taking more than $90 \%$ of their drug (as in previous studies with simvastatin in central nervous system diseases).$^{28} 29$

\section{Permitted comedications}

Medication that is contraindicated with simvastatin, and escitalopram or concomitant treatment with antidepressants other than escitalopram will not be permitted (see inclusion/exclusion criteria in online supplemental table S2). In case of current treatment with antidepressants at study entry, a wash-out of prior antidepressants for at least seven drug-free days prior to inclusion may be permitted (see inclusion criteria in online supplemental table S2). However, discontinuation of effective medication to enable study participation is prohibited. Other psychotropic drugs (including antipsychotics, anticonvulsants, lithium or St. John's Wort) are not permitted during the study period with the following exceptions: (1) in case of sleep difficulties, benzodiazepine or nonbenzodiazepine hypnotics (eg, zolpidem, zopiclone) will be allowed on an 'as needed basis'; (2) in case of acute anxiety or agitation, a benzodiazepine (preferably lorazepam) may be prescribed not exceeding $3 \mathrm{mg} / \mathrm{d}$ (other benzodiazepines at the equivalent dosage), (3) opiates for pain relief (see exclusion criteria in online supplemental table S2). Any clinical exacerbation requiring a higher benzodiazepine regimen or prescription of antipsychotics ('rescue medication') will be considered as a serious adverse event (SAE) (see 3.8 Safety and online supplemental material). In case of non-psychotropic medication, stable pharmacological medication for at least 14 days prior to study entry will be permitted. Patients with any changes in medication dose or frequency of therapy during the 14 days prior to study entry will not participate in the study (see inclusion criteria in online supplemental table S2). Concomitant use of statins will not be permitted (see exclusion criteria in online supplemental table S2).

\section{Sample size calculation}

So far, there are three small pilot RCTs in depressed patients (sample sizes ranging from $n=48$ to $n=68$ ) that have all found statistically significant beneficial effects of add-on statins versus placebo with large, clinically relevant effect sizes. A meta-analysis ${ }^{14}$ of these trials has found a standardised mean difference between add-on statins and add-on placebo of -0.73 (95\% CI -1.04 to -0.42 , $\mathrm{p}<0.001)$. However, it is well known that initial studies tend to overestimate the true effect size ${ }^{30}$ Therefore, we conservatively assume a standardised treatment difference (also known as Cohen's d) of 0.5, which is smaller than those reported by the three pilot RCTs. ${ }^{23} 3132$ Given a SD of 6-8 points on the MADRS, as commonly observed in RCTs in this population, ${ }^{33-35}$ a standardised effect size of 0.5 translates into a mean difference of 3-4 points, which is considered clinically relevant because it is about twice as large as the minimal clinically important difference (MCID) of 1.6-1.9 points that has been reported for the MADRS. ${ }^{36}$ Thus, a sample size of 64 patients per group results in a power of $80 \%$ for a comparison of the mean changes in MADRS score from baseline to week 12 between the two groups using a two-sample t-test at the usual two-sided level of $5 \%$. As the analyses will be adjusted for baseline scores, the actual power is likely to be higher; this will be confirmed in a blinded sample size review once $50 \%$ of the patients have reached week $12 .{ }^{37}$ Accounting for about 20\% dropout, we aim to recruit 80 patients per group (ie, 160 patients in total). Based on sex-specific prevalence rates and recruitment in our earlier studies, ${ }^{389}$ we expect that about two-thirds of the sample will be women. This will allow sex-specific analyses.

As the analyses will be adjusted for baseline scores, the actual power is likely to be higher than $80 \%$. To confirm this in an interim analysis, a blinded sample size review will be carried out, once $50 \%$ of the patients have reached week 12. Based on blinded estimates of the mean square error and the dropout rate, the required sample size will be recalculated and the sample size will be adjusted up to a maximum total sample size of 240 patients, if necessary. ${ }^{37}$

\section{Outcomes}

As primary outcome variable, we will use the change in MADRS $^{40}$ score from baseline to week 12 . As secondary outcome variables, we will use MADRS response (defined as $50 \%$ MADRS score reduction from baseline), MADRS remission (defined as MADRS score $<10$ ) and MADRS MCID. Also, we will assess as secondary outcome variables change scores in patients' self-reported Beck Depression Inventory (BDI-II) as well as the MCID according to BDI. ${ }^{41}$ We will additionally determine the clinician's impression of the severity of illness (Clinical Global Impression scale-Severity of illness) ${ }^{42}$ the clinician's impression whether and to what extent symptoms have improved (Clinical Global Impression scale-Improvement), ${ }^{42}$ the patient's impression whether and to what extent symptoms have improved (Patients' Global Impression of Change Scale), social functioning (Social and Occupational Functioning Assessment Scale, SOFAS), quality of life (EuroQol-5 Dimensions-3 Levels Questionnaire), with a calculated $\mathrm{MCID}^{43}$ and change scores in high-density lipoprotein, low-density lipoprotein and total cholesterol. At selected sites, additional blood samples for assessment of immune function and cellular metabolism will be obtained and analysed in an exploratory fashion. For the characteristics and definitions of primary and secondary endpoints, see table 1 . The efficacy and other outcome assessments at screening, baseline and different follow-up visits are shown in table 2.

\section{Randomisation and blinding}

Subjects will be randomised 1:1 to simvastatin or placebo (SIM+ES vs PL+ES) based on a randomisation list provided 
Table 1 Characteristics and definitions of primary and secondary endpoints

\begin{tabular}{|c|c|c|c|c|c|}
\hline Outcome & Instrument & Rating & Domain & Exactly defined outcome & Variable \\
\hline Primary & MADRS $^{*} \dagger$ & Clinician-rated & Severity of depression & Mean change from baseline & Continuous \\
\hline $\begin{array}{l}\text { Secondary/efficacy } \\
\text { (exploratory) }\end{array}$ & MADRS $^{\star} \dagger$ & Clinician-rated & Severity of depression & $\begin{array}{l}\text { Percentage response } \\
\text { (>50\% reduction from } \\
\text { baseline) } \\
\text { Percentage remission } \\
\text { (MADRS score }<10 \text { post- } \\
\text { treatment) } \\
\text { Percentage minimal } \\
\text { clinically important } \\
\text { difference (MCID; change } \\
\text { from baseline score }>1.9 \text { ) }\end{array}$ & Dichotomous \\
\hline
\end{tabular}

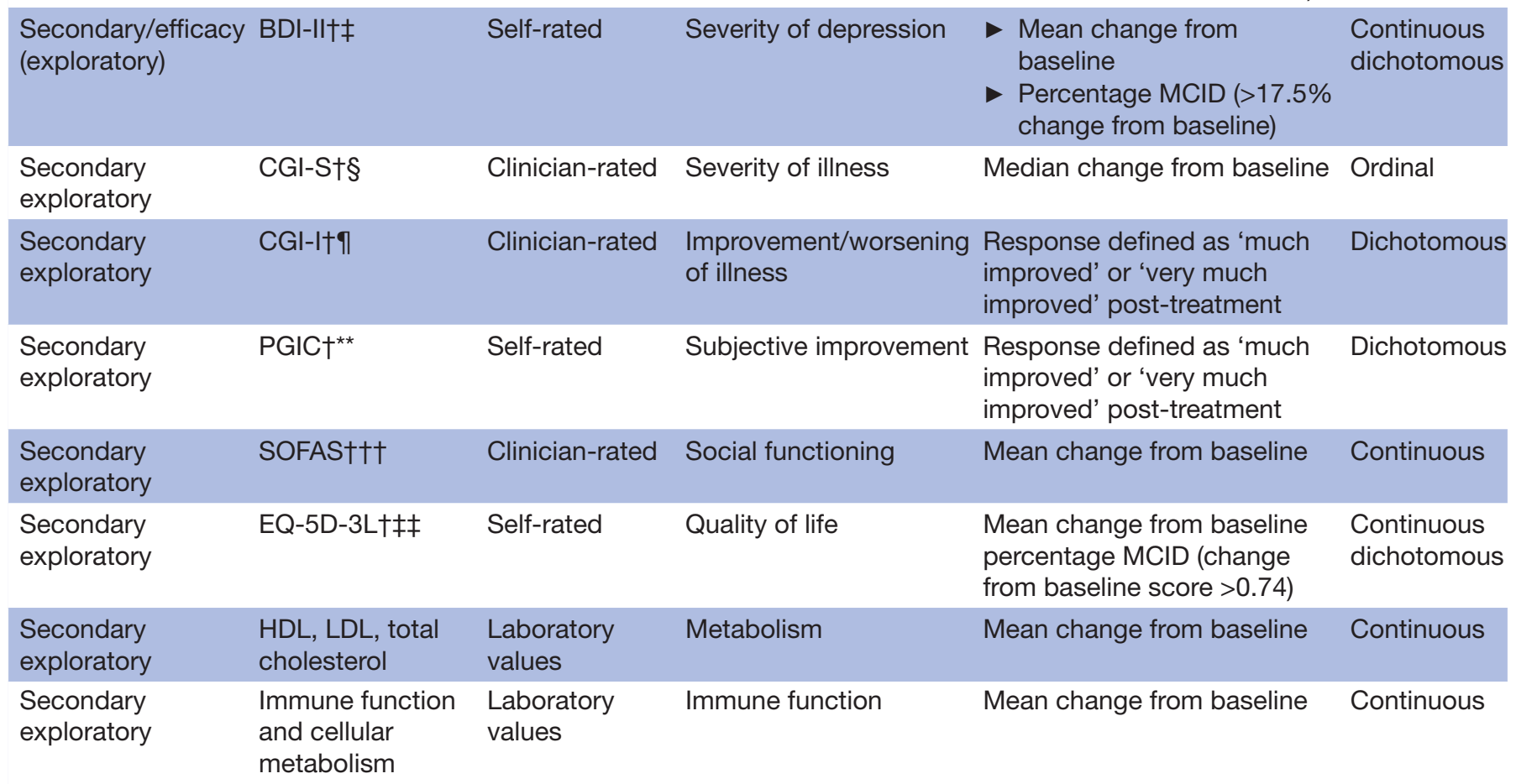

${ }^{*}$ The MADRS is a rating scale to measure depression severity. Each MADRS item is rated on a 0-6 scale. Total score ranges from 0 to 60 , where higher MADRS scores indicate higher levels of depressive symptoms.

†This instrument has been validated in German.

$\ddagger$ The Beck Depression Inventory-II is a 21-item validated instrument for the self-report of depressive symptoms, with individual item scores summed to yield a total possible BDI score that ranges from 0 to 63 . BDI scores from 0 to 13 suggest absent to minimal depressive symptoms, from 14 to 19 mild symptoms, from 20 to 28 moderate symptoms and from 29 to 63 severe symptoms.

$\S$ The CGI-S evaluates the severity of psychopathology on a scale of $0-7$. Considering total clinical experience, a participant is assessed on severity of mental illness at the time of rating according to: $0=$ not assessed; $1=$ normal (not at all ill); $2=$ borderline mentally ill; $3=$ mildly ill; $4=$ moderately ill; $5=$ markedly ill; $6=$ severely ill; $7=$ among the most extremely ill patients.

१The CGI-I is a clinician-rated instrument to measure clinicians' change in overall status on a scale ranging from 1 (very much improved) to 7 (very much worse).

**The PGIC is a participant-rated instrument to measure participant's change in overall status on a scale ranging from 1 (very much improved) to 7 (very much worse).

††The SOFAS is a rating scale used to determine social functioning on a scale ranging from 0 to 100 . A higher score represents better social and occupational functioning.

抽e EQ-5D-3L is a generic instrument of quality of life related to health. It contains five dimensions of health (mobility, personal care, daily activities, pain/discomfort and anxiety/depression) and each of them has three levels of seriousness (without problems, some problems or moderate problems and serious problems). The second part of the EQ-5D is a Visual Analogue Scale ranging from 0 (worse health status imaginable) to 100 (best imaginable health status). In it, the individual must mark the point in the vertical line that best reflects the assessment of their global health status today.

BDI-II, Beck-Depressions-Inventar II; BMI, body mass index; CGI-I, Clinicians' Global Impression of Improvement; CGI-S, Clinicians' Global Impression of Severity of illness; EQ-5D-3L, Generic Quality of Life Questionnaire; MADRS, Montgomery-Åsberg Depression Scale; MINI, Mini International Neuropsychiatric Interview; PGIC, Patient Global Impression of Change; SOFAS, Social and Occupational Functioning Assessment Scale. 
Table 2 Visit and documentation schedule

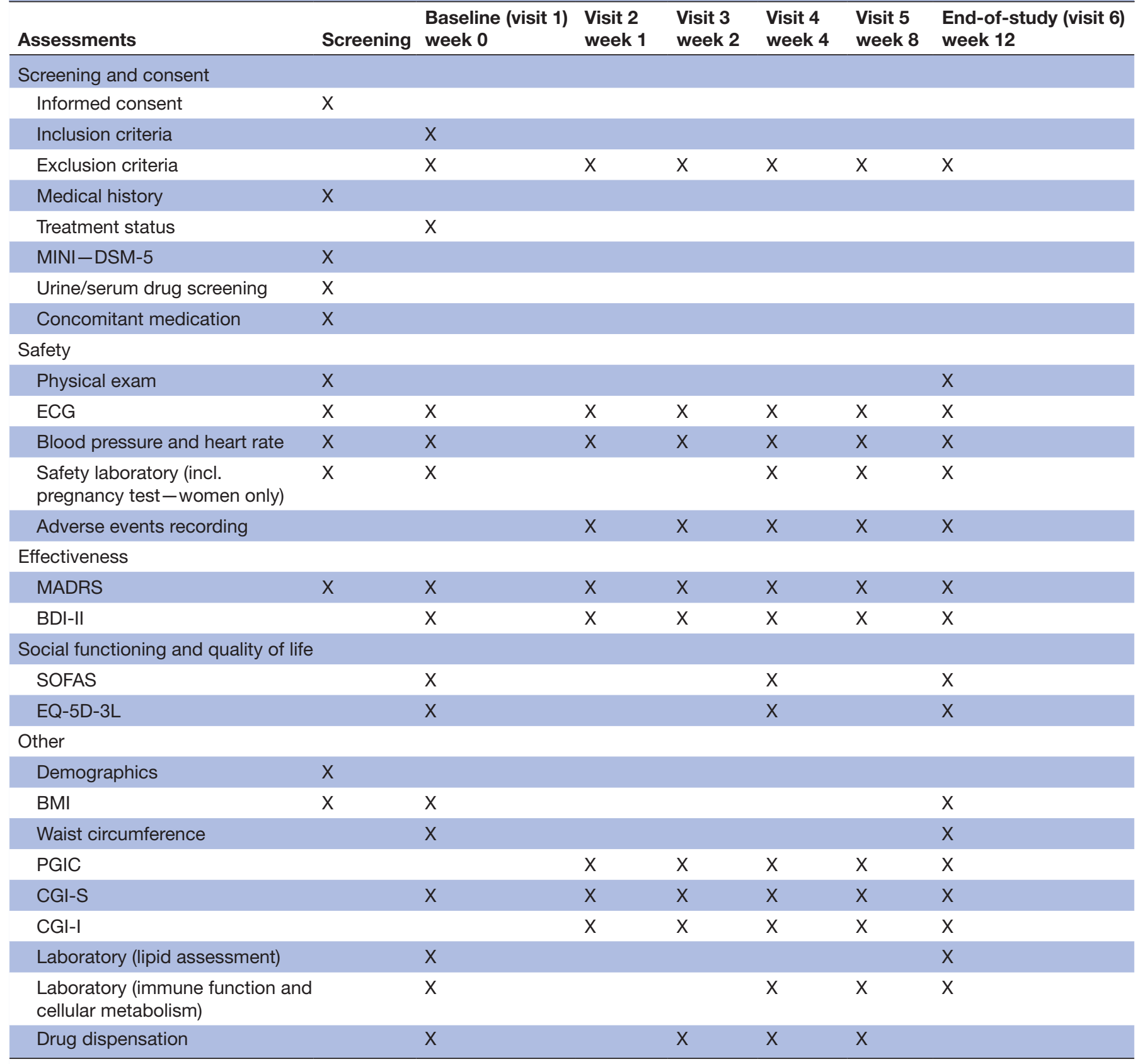

BDI-II, Beck-Depressions-Inventar II; BMI, Body-Mass-Index; CGI-I, Clinicians' Global Impression of Improvement; CGI-S, Clinicians' Global Impression of Severity of illness; EQ-5D-3L, Generic Quality of Life Questionnaire; MADRS, Montgomery-Åsberg Depression Scale; MINI, Mini International Neuropsychiatric Interview; PGIC, Patient Global Impression of Change; SOFAS, Social and Occupational Functioning Assessment Scale.

by the trial statistician. The randomisation will be based on a permuted block procedure stratified by study site.

Based on the randomisation codes, the pharmacy will centrally provide for each centre sequentially numbered, tamper-proof container, which are equal in weight and similar in appearance containing simvastatin or placebo. The pharmacy keeps the randomisation list unavailable to investigators/subinvestigators and the sponsor/sponsor representative until the end of study. Investigators/subinvestigators and patients will be blinded to the treatment. Simvastatin is an effective serum lipid-lowering drug that can decrease lipid levels. Particularly, LDL cholesterol is expected to be lower in the SIM+ES group. In order to ensure continued blinding, lipid values will be assessed only at V1 and the last trial visit (V6) to minimise the risk for unblinding of investigators/subinvestigators by revealing the lipid profile. At the same time, not assessing the lipid status of patients during the entire study participation would not represent current 
practice where lipid status is an important part of cardiovascular risk assessment. No unblinding of the investigators/subinvestigators due to specific side effects of simvastatin treatment is to be expected.

In case of an emergency, the blinding will be broken immediately, and the patient will receive immediate medical care. The local investigator will be responsible for deciding if knowledge of the group assignment is required for the medical care of the patient and initiate the unblinding procedure. Emergency envelopes for the unblinding are enclosed in the packages sent to the investigator site and are stored by the local investigator. When emergency envelopes are opened prematurely, date, time, reason for opening and name of the person opening have to be documented. After unblinding, the treatment of the individual participant will be stopped immediately; however, the participant will not be excluded from the analysis (see online supplemental material for more information on premature termination of the individual participant). An identical copy of the emergency envelopes for the unblinding will be sent and stored securely at the coordinating site (Charité-Universitätsmedizin Berlin). This way the coordinating staff is additionally available for immediate unblinding.

\section{Safety}

Simvastatin and escitalopram are approved for several indications since decades and have an established safety profile. All depressed patients enrolled in the trial will be continuously treated with a gold standard antidepressant (escitalopram) as described by the German National Disease Management Guideline ('Nationale Versorgungsleitlinie') for the treatment of MDD. In case of adverse reactions related to escitalopram, we will allow dose adjustments such as temporary dose reduction from 10 to $5 \mathrm{mg} / \mathrm{d}$ or from 20 to $10 \mathrm{mg} / \mathrm{d}$ for the duration of the adverse reaction. See table 2 for an overview of safety assessments at screening, baseline and different follow-up visits. We list the risks and detailed safety considerations for both medications, as well as definitions for adverse event (AE), serious $\mathrm{AE}$ and Suspected Unexpected Serious Adverse Reactions (SUSAR) in the online supplemental material.

All (S)AEs and SUSARs will be treated appropriately. Such treatment may include changes in study drug treatment including possible interruption or discontinuation, starting or stopping concomitant treatments, changes in the frequency or nature of assessments, hospitalisation or any other medically required intervention. Standard procedures for reporting of (S)AEs and SUSARs will be used.

For specifications for premature termination of the individual participant and the clinical study, see online supplemental material.

\section{Data management}

Patient related data will be recorded in study files (source data) under the participant's real name. The information required by the protocol will be entered onto the electronic case report forms (eCRF-secuTrial) under a pseudonym. Every patient will receive a patient number/ pseudonym which will be unique for this individual patient. Data required for the analysis will be acquired and transferred electronically to a central database at the Coordinating Center for Clinical Studies of Charité by means of the electronic data capture system secuTrial). The trial data will be stored digitally on a remote server with daily backups. After end of study, all data will be exported for checks of consistency and plausibility. After performing the checks, the data matrix will be transferred in pseudonymised form for the statistical evaluation. The statistical analysis and the biometrical report will be provided by the biostatistician in cooperation with the PI.

\section{Data analysis plan}

The primary analysis population will be the intention-totreat (ITT) population. The ITT population will include all randomised patients. We will repeat the analyses in the per-protocol sample. The primary analysis will compare MADRS changes from baseline to week 12 between SIM+ES and PL+ES by Gaussian linear models for repeated measures (so-called MMRM) with intervention, centre, time (week 1, 2, 4, 8 and 12), and interventionby-time interaction as factors and baseline MADRS score as covariate. The error terms are assumed to follow a multivariate normal distribution with unstructured covariance. Least squares mean changes from baseline will be reported for both groups with $95 \%$ CI as well as the difference between the least squares SIM+ES group means with 95\% CI and p-value testing the null hypothesis of no treatment effect. Although the model described above is robust to a certain extend to missing data, sensitivity analyses will be performed as supporting analyses including multiple imputation and last-observation-carried-forward. The latter will mainly be performed to facilitate comparison with previous trials.

The analyses of all secondary endpoints and safety parameters will have an exploratory character and will therefore not be adjusted for multiple testing. The secondary efficacy endpoints MADRS-response and MADRS-remission, and MADRS-MCID within 12 weeks will be analysed by logistic regression models with intervention and centre as factors and baseline MADRS as covariate. The treatment effects will be reported as ORs with 95\% CI and p-values testing the null hypotheses of no treatment effect. The analyses of continuous secondary efficacy endpoint as well as SOFAS, and EQ-5D-3L will follow the same lines as the primary outcome. All details of the statistical analyses including definitions of the analyses populations will be specified in the statistical analysis plan, which will be finalised before database lock and unblinding. All statistical analyses will be carried out using SAS software or the $\mathrm{R}$ package.

\section{Patient and public involvement}

A patient representative from the German Depression League ('Deutsche Depres-sionsliga'), the umbrella organisation of depression self-help groups in Germany 
was involved during the development of the study protocol. She will also be part of the Data and Safety Monitoring Board (DSMB) to represent the patient perspective.

\section{ETHICS AND DISSEMINATION}

This trial will be conducted in accordance with the current guidelines of International Conference on Harmonisation-Good Clinical Practice (ICH-GCP). GCP is an international ethical and scientific quality standard for designing, conducting, recording and reporting trials that involve the participation of human subjects. Compliance with this standard provides public assurance that the rights, safety and well-being of trial subjects are protected, consistent with the principles that have their origin in the Declaration of Helsinki, and that the clinical trial data are credible. Informed consent will be obtained for screening and for participation in the study. Study protocol, patient information and consent form have been approved by the relevant Ethics Committees and the relevant federal authority (Bundesinstitut für Arzneimittel und Medizinprodukte-BfArM). The Ethics Committee will immediately be informed (by the sponsor/sponsor representative) of all changes to the protocol (according to GCP-V $\S 10$ ) and of all events that could affect a patient's safety. The Ethics Committee will also be informed of all suspected SUSARs and of regular or premature termination of the study. We prospectively registered our study in the clinicaltrials.gov database and German Clinical Trials Register. The study results will be published irrespective of the study outcome in peer-reviewed journals and at (inter-)national conferences.

\section{Study management}

The sponsor/sponsor representative of the clinical study will have overall responsibility for the study. The PI together with each investigator of the study site will take clinical responsibility for the research team at each site. To enhance monitoring of study, we will seek advice from a DSMB comprised of methods and clinical experts in psychiatry and one patient representative. Thereby, the patients' perspective will be present at all DSMB meetings. Further details about DSMB including its charter are available on request.

Authorised representatives of the sponsor, a regulatory authority or an Independent Ethics Committee may visit the centre to perform audits or inspections, including source data verification. The purpose of a sponsor audit or inspection is to systematically and independently examine all study related activities and documents to determine whether these activities were conducted, and data were recorded, analysed and accurately reported according to the protocol, GCP, ICH guidelines and any applicable regulatory requirements.

\section{Perspectives}

According to a meta-analysis of small, RCTs, a beneficial antidepressive effect of simvastatin is to be expected. ${ }^{14}$
Many depressed patients with comorbid obesity might additionally benefit from simvastatin in terms of primary prevention of cardiovascular disease. The potential benefits of the study by far outweigh the risks associated with escitalopram and simvastatin. If successful, our trial would have immediate impact on clinical practice because escitalopram and simvastatin are available as inexpensive generic drugs with established safety.

\section{Ethics approval}

The study has been approved by a German ethics committee - Ethik-Kommission des Landes Berlin (Reference: 19/0226 - EK 11) on 22 January 2020.

\section{Author affiliations}

${ }^{1}$ Department of Psychiatry and Psychotherapy, Charité - Universitätsmedizin Berlin, corporate member of Freie Universität Berlin, Humboldt-Universität zu Berlin, and Berlin Institute of Health, Berlin, Germany

${ }^{2}$ NeuroCure Clinical Research Center, Charité - Universitätsmedizin Berlin, corporate member of Freie Universität Berlin, Humboldt-Universität zu Berlin, and Berlin Institute of Health, Berlin, Germany

${ }^{3}$ Clinical Research Unit, Charité - Universitätsmedizin Berlin, corporate member of Freie Universität Berlin, Humboldt-Universität zu Berlin, and Berlin Institute of Health, Berlin, Germany

${ }^{4}$ Department of Psychiatry and Psychotherapy, University Hospital of Leipzig, Leipzig, Germany

${ }^{5}$ Department of Psychiatry and Psychotherapy, University Medicine Greifswald, Greifswald, Germany

${ }^{6}$ Department of Psychiatry, Psychosomatics and Psychotherapy, Hospital of the Goethe University Frankfurt, Frankfurt am Main, Hessen, Germany

${ }^{7}$ Department of Psychosomatic Medicine, Center for Internal Medicine and Dermatology, Charité - Universitätsmedizin Berlin, corporate member of Freie Universität Berlin, Humboldt-Universität zu Berlin, and Berlin Institute of Health, Berlin, Germany

${ }^{8}$ Department of Psychiatry and Psychotherapy, Medical University of Luebeck, Luebeck, Germany

${ }^{9}$ Department of Psychiatry, Social Psychiatry and Psychotherapy, Hannover Medical School, Hannover, Germany

${ }^{10}$ Department of Psychiatry and Psychotherapy, University Medical Center HamburgEppendorf, Hamburg, Germany

${ }^{11}$ Department of Psychiatry, Psychotherapy and Psychosomatic Medicine, Hospital of the Goethe University Frankfurt, Frankfurt am Main, Germany

${ }^{12}$ Department of Medical Statistics, University Medical Center Göttingen, Gottingen, Germany

${ }^{13}$ Institute of Neuroimmunology and Multiple Sclerosis (INIMS), Center for Molecular Neurobiology, University Medical Center Hamburg-Eppendorf, Hamburg, Germany

\section{TwitterTim Friede @tim_friede and Stefan M Gold @GoldLabCharite}

Acknowledgements We are grateful to NeuroCure Clinical Research Center (NCRC), funded by the Deutsche Forschungsgemeinschaft (DFG, German Research Foundation) under Germany's Excellence Strategy-EXC-2049390688087, EXC257-39052203 and Berlin Institute of Health (BIH) for the help with study approvals and preparing the study protocol. We also thank the patient representative for her help with designing the study. We acknowledge support from the German Research Foundation (DFG) and the Open Access Publication Funds of Charité - Universitätsmedizin Berlin.

Contributors CO designed the study, obtained funding, developed protocol and study materials, obtained necessary approvals for the study and critically revised this manuscript. WRC contributed to protocol and study materials and drafted the manuscript. JN contributed to study materials. SM and SL helped with study approvals and preparing the study protocol. MK, DoP, SR, BE, HJG, UH, KH, TH, DJ, KJ, KGK, JPK, THCK, GL, DaP, AR, DS, MS, SS and AW contributed to study design and critically revised the manuscript. TF contributed to study design, obtained funding, developed the protocol and revised this manuscript. SMG contributed to study design, obtained funding, developed protocol and study materials and critically revised this manuscript. 
Funding This work is supported by the German ministry of education and research (BMBF). Grant number: 01KG1813. The study is sponsored by Charité Universitätsmedizin Berlin.

Competing interests $\mathrm{CO}$ reports personal fees from Allergan, Ferring, Fortbildungskolleg, Limes Klinikgruppe, Lundbeck, MedOnline, Medical Tribune, Neuraxpharm, SAGE Therapeutics and Stillachhaus outside the submitted work and reports grants from the German Research Foundation (DFG), German Ministry of Education and Research (BMBF), the European Union (Innovative Medicines Initiative) and the Brain \& Behavior Foundation (NARSAD). MK participates in the Berlin Institute of Health - Charité Clinician Scientist Program funded by the Charité-Universitätsmedizin Berlin and the Berlin Institute of Health. SM reports grants from Deutsche Forschungsgemeinschaft (DFG, German Research Foundation) during the conduct of the study. HJG reports grants and personal fees from Fresenius Medical Care, personal fees from Neuraxpharm, Servier, Janssen Cilag and grants from German Research Foundation (DFG), German Ministry of Education and Research (BMBF), Damp Foundation, European Union Joint Programme Neurodegenerative Disorders (JPND) and European Social Fund (ESF) outside the submitted work. UH reports personal fees from Janssen and Servier outside the submitted work. TF reports grants and non-financial support from German Ministry of Education and Research (BMBF) during the conduct of the study. KGK reports personal fees and other from Eli Lilly, Servier and Neuraxpharm, and grants, personal fees and other from Ferrer outside the submitted work. JPK reports grants and personal fees from Servier, personal fees from Springer, Hogrefe, Elsevier and Beltz outside the submitted work. THCK reports grants from German Ministry of Education and Research (BMBF) during the conduct of the study and personal fees from Allergan, Lundbeck, Otsuka, Trommsdorf, Novartis and Schwabe outside the submitted work. AR reports grants and personal fees from Medice, personal fees from Shire/Takeda, Janssen, Neuraxpharm, Servier and SAGE outside the submitted work. DS reports honoraria for lectures from or has been an advisor to Janssen, Lundbeck, Otsuka Pharma, Medice and Takeda. TF reports personal fees from Novartis, Bayer, Janssen, SGS, Roche, Boehringer Ingelheim, DaiichiSankyo, Galapagos, Penumbra, Parexel, Vifor, BiosenseWebster, CSL Behring, Fresenius Kabi, Coherex Medical and LivaNova outside the submitted work. SMG reports grants from German Ministry of Education and Research (BMBF), German Research Foundation (DFG), Federal Ministry of Health (BMG), Biogen and National Multiple Sclerosis Society (NMSS) during the conduct of the study and personal fees from Almirall S.A., Mylan, Celgene and FomF outside the submitted work. WRC, JN, DoP, SR, SL,SS, BE, KH, DJ, KJ, GL, DaP, MS and AW have nothing to disclose.

Patient consent for publication Not required.

Provenance and peer review Not commissioned; externally peer reviewed.

Supplemental material This content has been supplied by the author(s). It has not been vetted by BMJ Publishing Group Limited (BMJ) and may not have been peer-reviewed. Any opinions or recommendations discussed are solely those of the author(s) and are not endorsed by BMJ. BMJ disclaims all liability and responsibility arising from any reliance placed on the content. Where the content includes any translated material, BMJ does not warrant the accuracy and reliability of the translations (including but not limited to local regulations, clinical guidelines, terminology, drug names and drug dosages), and is not responsible for any error and/or omissions arising from translation and adaptation or otherwise.

Open access This is an open access article distributed in accordance with the Creative Commons Attribution Non Commercial (CC BY-NC 4.0) license, which permits others to distribute, remix, adapt, build upon this work non-commercially, and license their derivative works on different terms, provided the original work is properly cited, appropriate credit is given, any changes made indicated, and the use is non-commercial. See: http://creativecommons.org/licenses/by-nc/4.0/.

\section{ORCID iDs}

Christian Otte http://orcid.org/0000-0002-4051-997X

Woo Ri Chae http://orcid.org/0000-0002-6326-8333

Stefanie Märschenz http://orcid.org/0000-0003-3303-5819

Jan Philipp Klein http://orcid.org/0000-0001-9882-2261

Tim Friede http://orcid.org/0000-0001-5347-7441

Stefan M Gold http://orcid.org/0000-0001-5188-4799

\section{REFERENCES}

1 Abajobir AA, Abate $\mathrm{KH}$, Abbafati C, et al. Global, regional, and national incidence, prevalence, and years lived with disability for 328 diseases and injuries for 195 countries, 1990-2016: a systematic analysis for the global burden of disease study 2016. Lancet 2017;390:1211-59.

2 Whiteford HA, Degenhardt L, Rehm J, et al. Global burden of disease attributable to mental and substance use disorders: findings from the global burden of disease study 2010. Lancet 2013;382:1575-86.

3 Bromet E, Andrade LH, Hwang I, et al. Cross-National epidemiology of DSM-IV major depressive episode. BMC Med 2011;9:90.

4 Laursen TM, Musliner KL, Benros ME, et al. Mortality and life expectancy in persons with severe unipolar depression. J Affect Disord 2016;193:203-7.

5 GBD 2015 Obesity Collaborators, Afshin A, Forouzanfar MH, et al. Health effects of overweight and obesity in 195 countries over 25 years. N Engl J Med 2017;377:13-27.

6 NCD Risk Factor Collaboration (NCD-RisC). Worldwide trends in body-mass index, underweight, overweight, and obesity from 1975 to 2016: a pooled analysis of 2416 population-based measurement studies in 128.9 million children, adolescents, and adults. Lancet 2017;390:2627-42

7 Truthmann J, Mensink GBM, Bosy-Westphal A, et al. Metabolic health in relation to body size: changes in prevalence over time between 1997-99 and 2008-11 in Germany. PLoS One 2016;11:e0167159.

8 Global BMI Mortality Collaboration. Body-Mass indexand allcause mortality: individual-participant-data meta-analysis of 239 prospective studies in fourcontinents. Lancet 2016;388:776-86.

9 Lim SS, Vos T, Flaxman AD, et al. A comparative risk assessment of burden of disease and injury attributable to 67 risk factors and risk factor clusters in 21 regions, 1990-2010: a systematic analysis for the global burden of disease study 2010. Lancet 2012;380:2224-60.

10 Luppino FS, de Wit LM, Bouvy PF, et al. Overweight, obesity, and depression: a systematic review and meta-analysis of longitudinal studies. Arch Gen Psychiatry 2010;67:220-9.

11 Robinson JG, Stone NJ. The 2013 ACC/AHA guideline on the treatment of blood cholesterol to reduce atherosclerotic cardiovascular disease risk: a new paradigm supported by more evidence. Eur Heart J 2015;36:2110-8.

12 McFarland AJ, Anoopkumar-Dukie S, Arora DS, et al. Molecular mechanisms underlying the effects of statins in the central nervous system. Int J Mol Sci 2014;15:20607-37.

13 Parsaik AK, Singh B, Murad MH, et al. Statins use and risk of depression: a systematic review and meta-analysis. J Affect Disord 2014;160:62-7

14 Salagre E, Fernandes BS, Dodd S, et al. Statins for the treatment of depression: a meta-analysis of randomized, double-blind, placebocontrolled trials. J Affect Disord 2016;200:235-42.

15 Köhler O, Gasse C, Petersen L, et al. The effect of concomitant treatment with SSRIs and statins: a population-based study. Am J Psychiatry 2016;173:appiajp201615040463:807-15.

16 Otte C, Gold SM, Penninx BW, et al. Major depressive disorder. Nat Rev Dis Primers 2016;2:16065.

17 Gold SM, Köhler-Forsberg O, Moss-Morris R, et al. Comorbid depression in medical diseases. Nat Rev Dis Primers 2020;6:69.

18 Kloiber S, Ising M, Reppermund S, et al. Overweight and obesity affect treatment response in major depression. Biol Psychiatry 2007;62:321-6.

19 Puzhko S, Aboushawareb SAE, Kudrina I, et al. Excess body weight as a predictor of response to treatment with antidepressants in patients with depressive disorder. J Affect Disord 2020;267:153-70.

20 Chan A-W, Tetzlaff JM, Altman DG, et al. Spirit 2013 statement: defining standard protocol items for clinical trials. Ann Intern Med 2013;158:200-7.

21 European Medicines Agency. Guideline on clinical investigation of medicinal products in the treatment of depress, 2013. Available: http://www.ema.europa.eu/docs/en_GB/document_library/ Scientific_guideline/2013/05/WC500143770.pdf [Accessed 2 Sep 2016].

22 Sheehan DV, Lecrubier Y, Sheehan KH, et al. The Mini-International neuropsychiatric interview (M.I.N.I.): the development and validation of a structured diagnostic psychiatric interview for DSM-IV and ICD10. J Clin Psychiatry 1998;59 Suppl 20:22-33.

23 Gougol A, Zareh-Mohammadi N, Raheb S, et al. Simvastatin as an adjuvant therapy to fluoxetine in patients with moderate to severe major depression: a double-blind placebo-controlled trial. $J$ Psychopharmacol 2015;29:575-81.

24 Abbasi SH, Mohammadinejad P, Shahmansouri N, et al. Simvastatin versus atorvastatin for improving mild to moderate depression in post-coronary artery bypass graft patients: a double-blind, placebocontrolled, randomized trial. J Affect Disord 2015;183:149-55.

25 Vuletic S, Riekse RG, Marcovina SM, et al. Statins of different brain penetrability differentially affect CSF PLTP activity. Dement Geriatr Cogn Disord 2006;22:392-8. 
26 U.S. Food and Drug. Available: http://www.fda.gov/ForConsumers/ ConsumerUpdates/ucm257884.htm

27 Bech P, Tanghøj P, Cialdella P, et al. Escitalopram dose-response revisited: an alternative psychometric approach to evaluate clinical effects of escitalopram compared to citalopram and placebo in patients with major depression. Int $J$ Neuropsychopharmacol 2004;7:283-90.

28 Chan D, Binks S, Nicholas JM, et al. Effect of high-dose simvastatin on cognitive, neuropsychiatric, and health-related quality-of-life measures in secondary progressive multiple sclerosis: secondary analyses from the MS-STAT randomised, placebo-controlled trial. Lancet Neurol 2017;16:591-600.

29 Chataway J, Schuerer N, Alsanousi A, et al. Effect of high-dose simvastatin on brain atrophy and disability in secondary progressive multiple sclerosis (MS-STAT): a randomised, placebo-controlled, phase 2 trial. Lancet 2014;383:2213-21.

30 Leon AC, Davis LL, Kraemer HC. The role and interpretation of pilot studies in clinical research. J Psychiatr Res 2011;45:626-9.

31 Ghanizadeh A, Hedayati A. Augmentation of fluoxetine with lovastatin for treating major depressive disorder, a randomized double-blind placebo controlled-clinical trial. Depress Anxiety 2013;30:1084-8.

32 Haghighi M, Khodakarami S, Jahangard L, et al. In a randomized, double-blind clinical trial, adjuvant atorvastatin improved symptoms of depression and blood lipid values in patients suffering from severe major depressive disorder. J Psychiatr Res 2014;58:109-14.

33 Cipriani A, Koesters M, Furukawa TA, et al. Duloxetine versus other anti-depressive agents for depression. Cochrane Database Syst Rev 2012;10:CD006533.

34 Galizia I, Oldani L, Macritchie K, et al. S-Adenosyl methionine (same) for depression in adults. Cochrane Database Syst Rev 2016;10:CD011286.
35 Koesters M, Ostuzzi G, Guaiana G, et al. Vortioxetine for depression in adults. Cochrane Database Syst Rev 2017;7:CD011520.

36 Duru G, Fantino B. The clinical relevance of changes in the Montgomery-Asberg depression rating scale using the minimum clinically important difference approach. Curr Med Res Opin 2008;24:1329-35.

37 Friede T, Kieser M. Blinded sample size recalculation for clinical trials with normal data and baseline adjusted analysis. Pharm Stat 2011;10:8-13.

38 Otte $\mathrm{C}$, Hinkelmann K, Moritz S, et al. Modulation of the mineralocorticoid receptor as add-on treatment in depression: a randomized, double-blind, placebo-controlled proof-of-concept study. J Psychiatr Res 2010;44:339-46.

39 Otte C, Wingenfeld K, Kuehl LK, et al. Mineralocorticoid receptor stimulation improves cognitive function and decreases cortisol secretion in depressed patients and healthy individuals. Neuropsychopharmacology 2015;40:386-93.

40 Montgomery SA, Asberg M. A new depression scale designed to be sensitive to change. Br J Psychiatry 1979;134:382-9.

41 Button KS, Kounali D, Thomas L, et al. Minimal clinically important difference on the Beck Depression Inventory--II according to the patient's perspective. Psychol Med 2015;45:3269-79.

42 Guy W. ECDEU assessment manual for psychopharmacology. Rockville, MD: US Department of Health, Education, and Welfare Public Health Service Alcohol, Drug Abuse, and Mental Health Administration, 1976.

43 Walters SJ, Brazier JE. Comparison of the minimally important difference for two health state utility measures: EQ-5D and SF-6D. Qual Life Res 2005;14:1523-32. 